of the specimens, while resistance to moxifloxacin (S83I in ParC) was observed in $10.6 \%(9 / 85)$ of the specimens tested. Mutations predictive of dual resistance (both $23 \mathrm{~S}$ rRNA and ParC) were observed in $2.12 \%(4 / 189)$ of the specimens. Females 20-24 years had the highest prevalence $(18.3 \%$, $\mathrm{p}<0.001)$ of infection. In females, M. genitalium was significantly associated with C. trachomatis or N. gonorrhoeae/C. trachomatis $(p<0.001)$ co-infection. The prevalence of $M$. genitalium in a northern region was statistically significantly higher $(p=0.012)$, as compared to other regions in the province.

Conclusions The prevalence of M. genitalium (9.6\%) and associated resistance to azithromycin (63.6\%) in Saskatchewan is high, suggesting that empiric azithromycin therapy may not be adequate for treating these infections. A potential risk of azithromycin treatment failure could be avoided by performing resistance-guided therapy, as azithromycin is also a recommended drug for the treatment of $\mathrm{N}$. gonorrhoeae (combined with ceftriaxone) and C. trachomatis infections in Canada.

\section{P219 POLICY AND PROGRAM RECOMMENDATIONS FOR STBBI INCENTIVE-BASED TESTING IN HIGH-INCOME COUNTRIES: A SYSTEMATIC REVIEW}

1,2 J Finlay*, IJ Krahn, ${ }^{2} \mathrm{~T}$ Lambert, ${ }^{2} \mathrm{G}$ Meyer, ${ }^{2,3} \mathrm{~A}$ Singh, ${ }^{3} \mathrm{~V}$ Caine. ${ }^{1}$ University of Calgary, Calgary, Canada; ${ }^{2}$ Alberta Health Services, Edmonton, Canada; ${ }^{3}$ University of Alberta, Edmonton, Canada

\subsection{6/sextrans-2021-sti.306}

Background Despite increasing access to treatment and testing, rates of sexually transmitted and blood-borne infections (STBBI) continue to rise. At the same time, screening uptake remains suboptimal. Incentives (e.g. cash, gift cards, prize draws) have been proposed as a way to increase the immediate rewards of STBBI testing and facilitate a greater uptake of screening. This systematic review was conducted to determine if patient incentives increase STBBI screening uptake in highincome countries.

Methods Our review was performed according to the recommendations of the Preferred Reporting Items for Systematic Reviews and Meta-Analyses (PRISMA) statement and the Cochrane Handbook for Systematic Reviews of Interventions. MEDLINE, EMBASE, PsycINFO, CINAHL, Scopus, and Cochrane Library were searched from 2013 to 2020. Inclusion criteria were: English language, high-income countries, primary research studies, and age $>13$ years. Study quality was assessed using Joanna Briggs Institute quality assessment tools.

Results The search yielded 6217 abstracts.13 studies met the inclusion criteria. Of these studies, five were located in the United States, three in the United Kingdom, and five in Australia. Seven studies took place in the community, five studies took place in clinical settings, and one study utilized a combination of community and clinical settings. Study design and intervention heterogeneity precluded meta-analysis or data pooling. Populations screened included: post-secondary students, parolees or probationers, youth, and inner-city emergency department patients. Incentivized STBBI tested were HIV $(n=5)$, chlamydia $(n=7)$, and multiple infections $(n=1)$. Incentives offered were monetary (cash/gift cards/not specified) $(n=8)$, non-monetary $(n=2)$, and mixed $(n=3)$.

Conclusion Both monetary and non-monetary incentives enhance STBBI screening in high-income countries.
Incentivized testing programs are most effective when developed specific to context and target population. Further research is needed to analyze incentivized screening across similar study designs and to evaluate long-term effectiveness.

\section{P220 THE BURDEN OF HIV ON MALARIA AND SEXUALLY TRANSMITTED AND REPRODUCTIVE TRACT INFECTIONS AMONG PREGNANT WOMEN OF RURAL, ZAMBIA}

${ }^{1} E$ Chaponda*, ${ }^{2} \mathrm{R}$ Chico, ${ }^{2} \mathrm{~J}$ Bruce, ${ }^{1} \mathrm{C}$ Michelo, ${ }^{2} \mathrm{D}$ Chandramohan. 'University of Zambia, Lusaka, Zambia; ${ }^{2}$ London School of Hygiene and Tropical Medicine, London, UK

\subsection{6/sextrans-2021-sti.307}

Background Malaria and curable sexually transmitted and reproductive tract infections (STIs/RTIs) are important causes of adverse birth outcomes. HIV has been associated with STIs/ RTIs and malaria infection. The study objectives were to estimate the prevalence of malaria and STIs/RTIs and to identify risk factors for infection in rural Zambia.

Methods A total of 1,086 pregnant women were enrolled at antenatal care booking, socio-demographic information and biological samples were collected for diagnosis of malaria, STIs/RTIs and routine HIV testing.

Results Participants had a mean age of 25.6 years. The prevalence of HIV was $13.2 \%$ (95\% confidence interval [CI], 11.3, 15.3 ) and the highest burden was in multigravidae (15.9\%) followed by secundigravidae $(13.9 \%)$ and was lowest in primigravidae $(8.0 \%), \mathrm{P}=0.018$. Of 1084 women, 700 had at least one STI/RTI (95\% CI, 64.6\%; 95\%, 61.7, 67.4). The prevalence of STIs/RTIs was as follows: chlamydia $5.2 \%$, (95\% CI, 4.0, 6.6); gonorrhoea 3.1\% (95\% CI, 2.2, 4.4); trichomoniasis $24.8 \%$ (95\% CI, 22.3, 27.5); bacterial vaginosis 48.7\% (95\% CI, 45.2, 51.2) and syphilis 7.1\% (95\% CI, 5.6, 8.7). The malaria prevalence measured by PCR was $57.8 \%$ (95\% CI, 54.9, 60.8).

HIV infection was associated with having gonorrhoea, (aOR $=2.80 ; 95 \%$ CI: 1.25, 6.26); bacterial vaginosis $(\mathrm{aOR}=$ 2.41; 95\% CI: 1.66, 3.51); syphilis infection $(\mathrm{aOR}=2.56$; 95\% CI: 1.48, 4.41); having any STIs/RTIs $(\mathrm{aOR}=2.10$; $95 \% \mathrm{CI}: 1.39,3.19)$ and malaria infection $(\mathrm{aOR}=1.46$; 95\% CI 1.00-2.13, $\mathrm{P}=0.045)$.

Conclusion HIV infection plays an important role in the prevalence of malaria and STIs/RTIs although it's effect on malaria prevalence in this population was neutralised by its concentration in multigravidae who have acquired immunity against malaria. Preventive and control efforts against malaria and STIs/RTIs should be targeted at the groups that needs them most in resource limited settings.

\section{P221 SYNDROMIC MANAGEMENT OF CURABLE SEXUALLY TRANSMITTED AND REPRODUCTIVE TRACT INFECTIONS AMONG PREGNANT WOMEN IN RURAL ZAMBIA}

${ }^{1} E$ Chaponda*, ${ }^{2} \mathrm{R}$ Chico, ${ }^{2} \mathrm{~J}$ Bruce, ${ }^{1} \mathrm{C}$ Michelo, ${ }^{2} \mathrm{D}$ Chandramohan. ${ }^{1}$ University of Zambia, Lusaka, Zambia; ${ }^{2}$ London School of Hygiene and Tropical Medicine, London, UK

\subsection{6/sextrans-2021-sti.308}

Background This study estimated the prevalence of curable sexually transmitted and reproductive tract infections (STIs/ RTIs) among pregnant women attending antenatal care (ANC) in rural Zambia and evaluated the effectiveness of syndromic 
management of STIs/RTIs versus reference-standard laboratory diagnoses.

Methods This was an observational cross-sectional study of 1,086 pregnant women. Participants were enrolled at ANC booking, socio-demographic information and biological samples were collected, and the provision of syndromic management based care was documented. The Piot-Fransen model was used to evaluate the effectiveness of syndromic management versus etiological testing.

Results Participants had a mean age of 25.6 years and a mean gestational age of 22.0 weeks. Of 1084 women, 700 had at least one STI/RTI $(64.6 \%$; 95\% confidence interval [CI], 61.7, 67.4). Only $10.2 \%$ of infected women received any treatment for a curable STI/RTI (excluding syphilis). Treatment was given to 0 of 56 women with chlamydia (prevalence 5.2\%; $95 \%$ CI, 4.0, 6.6), $14.7 \%$ of participants with gonorrhoea (prevalence $3.1 \% ; 95 \%$ CI, 2.2, 4.4), 7.8\% of trichomoniasis positives (prevalence 24.8\%; 95\% CI, 22.3, 27.5); $7.5 \%$ of women with bacterial vaginosis (prevalence 48.7\%; 95\% CI, 45.2 , 51.2). An estimated 7.1\% (95\% CI, 5.6, 8.7) of participants had syphilis and received treatment.

Conclusions Curable STIs/RTIs were common and the majority of cases were undetected and untreated. Alternative approaches are urgently needed in the ANC setting in rural Zambia.

\section{\begin{tabular}{|l|l}
\hline P222 AWARENESS OF INTERNET-BASED TESTING FOR \\
\hline
\end{tabular} SEXUALLY-TRANSMITTED AND BLOOD-BORNE INFECTIONS AMONG SEXUAL MINORITY MEN IN BRITISH COLUMBIA, CANADA}

${ }^{1} A$ Ablona*, ${ }^{1,2} \mathrm{~A}$ Montiel, ${ }^{3} \mathrm{~B}$ Klassen, ${ }^{2} \mathrm{~K}$ Card, ${ }^{1,4}{ }^{4}$ lyamu, ${ }^{1} \mathrm{H}$ Chang, ${ }^{1} \mathrm{D}$ Haag, ${ }^{5} \mathrm{E}$ Korol, ${ }^{6} \mathrm{~S}$ Bannar-Martin, ${ }^{7} \mathrm{D}$ Brennan, ${ }^{7} \mathrm{D}$ Grace, ${ }^{2} \mathrm{C}$ Worthington, ${ }^{2,3} \mathrm{~N}$ Lachowsky, ${ }^{1,4} \mathrm{M}$ Gilbert. ${ }^{1} B C$ Centre For Disease Control, Vancouver, Canada; ${ }^{2}$ University of Victoria, Victoria, Canada; ${ }^{3}$ Community-Based Research Centre, Vancouver, Canada; ${ }^{4}$ University of British Columbia, Vancouver, Canada; ${ }^{5}$ Interior Health Authority, Kelowna, Canada; ${ }^{6}$ sland Health Authority, Victoria, Canada; ${ }^{7}$ University of Toronto, Toronto, Canada

\subsection{6/sextrans-2021-sti.309}

Background GetCheckedOnline is an internet-based testing program for HIV, Hepatitis C, and other sexually-transmitted infections (STIs) in British Columbia (BC), Canada. The program launched in 2014 in Vancouver, BC's largest urban centre, and expanded to six smaller urban and suburban BC communities in 2016. We sought to measure GetCheckedOnline awareness among gay, bisexual, and other men who have sex with men (gbMSM), and to identify characteristics that may contribute to program awareness.

Methods Sex Now was a cross-sectional online health survey of Canadian gbMSM aged $\geq 15$ years recruited from 10/ 2019-02/2020. BC residents were asked if they knew about GetCheckedOnline and additional questions related to implementation outcomes. We built a multivariable logistic regression model including all variables significant in univariate analysis to quantify associations with awareness, and report adjusted odds ratios and 95\% confidence intervals (AOR [95\% CI]).

Results Among participants living in regions where GetCheckedOnline is available ( $\mathrm{n}=1132$, median age: 36 years), $38.1 \%$ were aware of GetCheckedOnline. Greater GetCheckedOnline awareness was associated with living outside of Vancouver $(\mathrm{AOR}=2.15[1.50-3.10])$, identifying as queer $(\mathrm{AOR}=1.58$
[1.13-2.21]), having post-graduate education $\quad(\mathrm{AOR}=1.84$ [1.17-2.91]), being out to healthcare providers $(\mathrm{AOR}=1.97$ [1.22-3.24]), using $\geq 3$ geolocation-based sex-seeking apps $(\mathrm{AOR}=2.15$ [1.40-3.33]), and past-year involvement in LGBTQ2S+-specific activities (AOR=1.56 [1.10-2.23]). Awareness decreased with increasing years of age $(\mathrm{AOR}=0.98$ [0.97-1.00]). Compared with participants who usually tested for STIs at a sexual health clinic, awareness was lower among participants who usually tested for STIs through their family doctor $(\mathrm{AOR}=0.50[0.33-0.73])$ or at walk-in medical clinics $(\mathrm{AOR}=0.54[0.30-0.96])$, and among those who had never tested for STIs previously (AOR $=0.17$ [0.06-0.39]).

Conclusion Almost 40\% of gbMSM in our sample were aware of GetCheckedOnline. Increasing promotion to gbMSM who are not out to their healthcare provider, who have never tested previously for STIs, or who are less connected to LGBTQ2S + communities may improve program reach.

\section{P224 LONELINESS AMONG OLDER BLACK AND WHITE PEOPLE LIVING WITH HIV}

1J Berko*, IJ Berko, ${ }^{1} \mathrm{~T}$ Loo, ${ }^{2} \mathrm{~L}$ MacLaren, ${ }^{3} \mathrm{G}$ Huhn, ${ }^{4} \mathrm{~T}$ Nwafor, ${ }^{4} \mathrm{~F}$ Spinelli, ${ }^{4,5} \mathrm{~A}$ Zolopa. ${ }^{1}$ Mazonson and Santas, Inc., Larkspur, USA; ${ }^{2}$ Whitman-Walker Health, Washington, DC, USA; ${ }^{3}$ Cook County Health, Chicago, USA; ${ }^{4}$ ViiV Healthcare, Raleigh, USA; ${ }^{5}$ Stanford University, Stanford, USA

\subsection{6/sextrans-2021-sti.310}

Background Loneliness is a concern among older people living with HIV (PLWH), especially given the ongoing COVID-19 pandemic. Currently, there is little research characterizing loneliness and race. To investigate how HIV impacts older (age 50+) PLWH in the US, a study called Aging with Dignity, Health, Optimism and Community (ADHOC) was launched at ten sites to collect self-reported data. This study uses ADHOC data to compare loneliness between older Black and White PLWH.

Methods A cross-sectional analysis was performed to compare loneliness between older Black and White PLWH. Loneliness was assessed using the Three-item Loneliness Scale, a validated patient-reported outcome measure (range 3-9), with higher scores indicating greater loneliness. Student's t-test was used to compare loneliness between White and Black people. Control variables for loneliness were identified a priori and included in a multivariable linear regression model.

Results Of 868 participants, the average age was 60 (SD 6.2) years, $77 \% \quad(\mathrm{~N}=671)$ were White and 23\% ( $=197)$ were Black, $85 \%$ were male, and $82 \%$ were gay or lesbian. In bivariate analyses, Black participants were less lonely than White participants $(5.2$ vs $5.6, \mathrm{P}=.02)$. In the multivariable linear regression analysis, Black participants were again less lonely than Whites (coef. $-0.45, \mathrm{P}=0.01$ ) while controlling for age, education, depression, anxiety, number of co-morbid conditions, being single, income, gender, sexual orientation, and spirituality (Adjusted R2 $=0.38 ; \mathrm{P}<.01$ ).

Conclusion Among PLWH over age 50 in ADHOC, Black participants experienced less loneliness than White participants, even after controlling for a variety of factors. Additional research is needed to better understand the causes of these racial differences, so that programs designed to decrease loneliness among PLWH can consider the unique characteristics of each group. 